\title{
Disruption of a Retinal Guanylyl Cyclase Gene Leads to Cone- Specific Dystrophy and Paradoxical Rod Behavior
}

\author{
Ruey-Bing Yang, ${ }^{2}$ Susan W. Robinson, ${ }^{1}$ Wei-Hong Xiong, ${ }^{5}$ King-Wai Yau, ${ }^{5}$ David G. Birch, ${ }^{3,4}$ and \\ David L. Garbers ${ }^{1,2}$ \\ ${ }^{1}$ Howard Hughes Medical Institute and Departments of 2 Pharmacology and ${ }^{3}$ Ophthalmology, University of Texas \\ Southwestern Medical Center, Dallas, Texas 75235-9050, ${ }^{4}$ Retina Foundation of the Southwest, Dallas, Texas 75231, and \\ 5 Howard Hughes Medical Institute and Department of Neuroscience, Johns Hopkins University School of Medicine, \\ Baltimore, Maryland 21205
}

One of two orphan photoreceptor guanylyl cyclases that are highly conserved from fish to mammals, GC-E (or retGC1) was eliminated by gene disruption. Expression of the second retinal cyclase (GC-F) as well as the numbers and morphology of rods remained unchanged in GC-E null mice. However, rods isolated from such mice, despite having a normal dark current, recovered from a light flash markedly faster. Unexpectedly, the a- and b-waves of electroretinograms (ERG) from dark-adapted null mice were suppressed markedly. Cones, initially present in normal numbers in the retina, disappeared by 5 weeks, based on ERG and histology. Thus, the GC-E-deficient mouse defines a model for cone dystrophy, but it also demonstrates that morphologically normal rods display paradoxical behavior in their responses to light.

Key words: guanylyl cyclases; retina; photoreceptors; gene disruption; cone dystrophy; mice; cyclic GMP; guanylyl cyclase-E
Vision begins in retinal photoreceptors, where the photoisomerization of the visual pigment leads to a G-protein-mediated activation of a phosphodiesterase hydrolyzing cGMP. As a result, the intracellular cGMP level decreases, and cGMP-gated ion channels close to produce a membrane hyperpolarization (for review, see Lagnado and Baylor, 1992; Yarfitz and Hurley, 1994; Yau, 1994). The closure of these channels results in a decrease in the intracellular $\mathrm{Ca}^{2+}$ concentration, which is proposed to act via guanylyl cyclase-activating proteins (GCAP1 and GCAP2) to increase guanylyl cyclase activity (Palczewski et al., 1994; Dizhoor et al., 1995) (for review, see Polans et al., 1996). The increase in cyclase activity then would represent an important mechanism for photoreceptor adaptation and recovery.

Two eye-specific guanylyl cyclases have been identified in the mammal; these are designated RetGC-1 and RetGC-2 in human (Shyjan et al., 1992; Lowe et al., 1995) and guanylyl cyclase-E (GC-E) and GC-F in rat (Yang et al., 1995). They are members of the family of membrane receptor guanylyl cyclases, all of which contain an extracellular putative ligand-binding domain, an apparent single transmembrane segment, and intracellular protein kinase homology and cyclase catalytic domains (Garbers and Lowe, 1994). With respect to the putative ligand-binding domain, the retinal cyclases remain orphan receptors. That there are two

\footnotetext{
Received Dec. 9, 1998; revised April 27, 1999; accepted May 4, 1999.

This research was supported in part by a grant-in-aid of research from the National Academy of Sciences (D.L.G.), Sigma Xi, The Scientific Research Society (R.B.Y.), and the National Eye Institute (K.-W.Y and D.G.B., Grant EY05235). This work was supported by the Howard Hughes Medical Institute. We thank Dr. Gabriel Travis for providing $r d s$ /peripherin and rhodopsin antibodies, Valerie Mach and Kristen Rossi for assistance in maintaining the mouse colony, and Dr. Clint Makino for valuable help and advice on the recordings from single mouse rods.

Correspondence should be addressed to Dr. David L. Garbers, Howard Hughes Medical Institute, 5323 Harry Hines Boulevard, Dallas, TX 75235-9050.

Dr. Yang's present address: Department of Molecular Biology, Genentech Incorporated, 1 DNA Way, South San Francisco, CA 94080.

Copyright (C) 1999 Society for Neuroscience $0270-6474 / 99 / 195889-09 \$ 05.00 / 0$
}

eye-specific guanylyl cyclases, both containing apparent ligandbinding domains conserved across human, rat, cow, and fish (Seimiya et al., 1997), implies significant pressure to retain the nature of the domain, possibly for the purpose of ligand recognition.

The retinal guanylyl cyclases appear predominantly, but not exclusively, in the photoreceptor outer segment layer. In the rod-dominant rat retina, GC-E appears uniformly distributed in the outer segment layer (Cooper et al., 1995). In cat, monkey, and human retinas, which have a significant number of cones, the GC-E-like immunoreactivity appears greater in cone than in rod outer segments (Dizhoor et al., 1994; Liu et al., 1994; Cooper et al., 1995). In the cone-rich retina of chicken, GC-E immunostaining is strong and uniform across the outer segment layer (Cooper et al., 1995). The relative distribution of GC-F in the outer segment layer is not well documented. Based on double-label immunogold electron microscopy, GC-F appears to be coexpressed with GC-E in the same rod outer segment (Yang and Garbers, 1997). In monkey retina, in situ hybridization experiments indicate a uniform distribution of the message for GC-F across the outer segment layer, but it has not been established whether this cyclase is also present in cones (Lowe et al., 1995).

In this paper we report the disruption of the GC-E gene. We have found that mice lacking the gene have morphologically normal rods at least up to 1 year of age, but the cones rapidly degenerate. The rods have a normal dark current despite the absence of functional GC-E and no compensatory increase in the expression of GC-F.

\section{MATERIALS AND METHODS}

Generation of GC-E-deficient mice. The GC-E gene (Gucy2e) from the mouse strain $129 / \mathrm{SvJ}$ was used to construct a targeting vector. A neomycin-resistance gene cassette replaced a portion of exon 5, which codes for the transmembrane region, leaving a coding sequence that could yield only a truncated protein (see Fig. 1a). SM1 embryonic stem 
(ES) cells (from Dr. Robert Hammer, University of Texas Southwestern Medical Center, Dallas, TX) derived from the inbred 129/SvJ mouse line were cultured on an irradiated STO-LIF fibroblast feeder layer (Ramirez-Solis et al., 1993). ES cells $\left(1.0 \times 10^{7}\right)$ were electroporated at $0.23 \mathrm{kV}, 500 \mu \mathrm{FD}$ with $25 \mu \mathrm{g}$ of linearized targeting vector DNA. G418 $(180 \mu \mathrm{g} / \mathrm{ml})$ (Life Technologies, Gaithersburg, MD) and gancyclovir (1 $\mu \mathrm{M})$ were added to the medium for selection for 4-5 d, until ES cell colonies formed. To identify clones with targeted disruption of the GC-E gene, we expanded the ES cells and prepared genomic DNA for Southern blot analysis (see Fig. 1b). Chimeric mice were generated by injecting the GC-E-disrupted ES cells into C57BL/6J blastocysts that subsequently were implanted into the uteri of pseudopregnant foster females. Seven chimeras were born and mated with $\mathrm{C} 57 \mathrm{BL} / 6 \mathrm{~J}$ mice to test for germline competency of the targeted clone; one of these mice transmitted the mutant allele. ES cell-derived progeny were screened for the presence of the targeted allele by Southern blot (see Fig. 1b) and/or PCR analysis. Heterozygous animals were inbred to generate homozygous mice lacking a functional GC-E gene, verified by Southern blot analysis. The targeted allele has been maintained in both $129 / \mathrm{SvJ}$ and C57BL/6J backgrounds.

Western blot and histological analyses. For Western blotting the posterior portion of the eyecup was collected and homogenized in $1 \times \mathrm{Lae}-$ mmli buffer (62.5 mu Tris-HCl, pH 6.8, $2 \%$ SDS, $10 \%$ glycerol, $0.005 \%$ bromophenol blue, and 5\% 2-mercaptoethanol). Total proteins were separated by SDS-polyacrylamide gel electrophoresis, transferred to polyvinylidene fluoride (PVDF) membranes (Immobilon-P, Millipore, Bedford, MA), and probed with antisera against GC-E, GC-F, or $r d s /$ peripherin (1:1000 dilution) as described (Travis et al., 1991; Yang and Garbers, 1997). For histology the eyes were removed, fixed overnight at $4^{\circ} \mathrm{C}$ with a solution of $10 \%$ formalin, and embedded in paraffin. Sections of 3 or $4 \mu \mathrm{m}$ were cut through the optic nerve head and stained with hematoxylin and eosin. Cone cells were identified by their nuclear structure (Carter-Dawson and LaVail, 1979) or by labeling with peanut agglutinin (PNA) as described by Blanks and Johnson (1984). For PNA labeling, the sections were deparaffinized with xylene and rehydrated through an ethanol series. After being blocked with $500 \mu \mathrm{g} / \mathrm{ml}$ bovine serum albumin in PBS, the sections were incubated with $100 \mu \mathrm{g} / \mathrm{ml}$ biotin-conjugated PNA (Vector Laboratories, Burlingame, CA) for 20 min at room temperature. Nonspecific binding was determined by incubation with biotin-PNA plus $50 \mathrm{~mm}$ D-galactose (Sigma, St. Louis, MO). Labeling was detected with the Vectastain ABC peroxidase kit (Vector Laboratories), followed by diaminobenzidine substrate. Sections were counterstained with methyl green.

Electroretinogram (ERG) recordings. Full-field corneal ERGs were obtained in a Ganzfeld dome from mice of indicated genotypes and ages. After at least $12 \mathrm{hr}$ of dark adaptation, the mice were anesthetized with ketamine $(200 \mathrm{mg} / \mathrm{kg})$ and xylazine $(10 \mathrm{mg} / \mathrm{kg})$. Pupils were dilated with local cyclopentolate hydrochloride drops before study. A gold wire coil placed on one cornea was referenced to a similar gold wire in the mouth. A needle electrode in the tail served as a ground. Signals were amplified 10,000-fold with a Tektronix (Beaverton, OR) AM502 differential amplifier ( $3 \mathrm{~dB}$ down at 2 and 10,000 $\mathrm{Hz}$ ), digitized (sampling rate 1.25-5 $\mathrm{kHz}$ ), and averaged on a personal computer. Two different flash stimulators were used. A Grass photostimulator provided $10 \mathrm{msec}$, shortwavelength flashes (Wratten 47A: $\lambda_{\max }=470 \mathrm{~nm}$; half-bandwidth $=55$ $\mathrm{nm}$ ) from -3.0 to $-1.0 \log$ scotopic troland-seconds (scot td-sec) in 0.3 $\log$ unit steps. A high-intensity flash unit (Novatron, Dallas, TX) produced 1.3 msec, short-wavelength flashes (Wratten W47B: $\lambda_{\max }=449$ $\mathrm{nm}$; half-bandwidth $=47 \mathrm{~nm}$ ) from 1.0 to $3.4 \log$ scot td-sec in $0.3 \log$ unit steps.

The leading edge of the rod a-wave was fit by the Lamb and Pugh (1992) model for the activation phase of phototransduction, which gives:

$$
P 3(i, t) \cong\left[1-\exp \left(-i \cdot S\left(t-t_{\mathrm{d}}\right)^{2}\right)\right] R m P 3 \text { for } t>t_{\mathrm{d}}
$$

where $P 3$ is the sum of the responses of individual rods. The amplitude of $P 3$ is a function of flash intensity $(i)$ and time $(t)$ after flash onset. $S$ is a sensitivity parameter that scales $i$. RmP3 is the maximum response, and $t_{\mathrm{d}}$ is a brief delay.

In the double-flash experiments two Novatron flash units were used within the full-field dome. A $1.5 \mathrm{log}$ scot td-sec test flash was followed by a $3.4 \log$ scot td-sec probe flash. The degree of recovery of the a-wave at various times $t$ after the test flash was calculated from the size of the a-wave evoked by the probe flash divided by that evoked by the probe flash in the absence of the test flash. The recovery time course was fit by an exponential decline function:

$$
\exp [-(t-T) / \tau]
$$

where $T$ is the critical delay before any response decline begins and $\tau$ is the decline time constant.

Single-cell electrophysiology. The procedures were primarily according to Sung et al. (1994). GC-E null or wild-type mice aged 8-10 weeks were dark-adapted overnight. Animals were killed by $\mathrm{CO}_{2}$ asphyxiation under dim red light. All subsequent procedures were performed under infrared light. The retina was isolated from the enucleated eye in chilled, oxygenated Leibovitz's L-15 medium (Life Technologies) and placed photoreceptor-side up on a glass capillary array (10- $\mu \mathrm{m}$-diameter capillaries; Galileo Electro-Optics, Sturbridge, MA) on which the retina was held by suction, allowing the vitreous humor to be removed by moving a razor blade between the retina and the array. The retinal pieces were stored in L-15 medium on ice until use. When needed, a piece of retina was chopped under L-15 medium containing $8 \mu \mathrm{g} / \mathrm{ml}$ deoxyribonuclease (Sigma) with a razor blade mounted on a lever arm, and a suspension of small retinal fragments was transferred into the recording chamber. The chamber temperature was held at $36-38^{\circ} \mathrm{C}$ by perfusing it continuously with heated solution buffered with bicarbonate and bubbled with $95 \%$ $\mathrm{O}_{2} / 5 \% \mathrm{CO}_{2}$, pH 7.4. The outer segment of an isolated rod or a rod projecting from a small fragment of retina was drawn into a suction electrode connected to a current-to-voltage converter. The recorded membrane current was filtered with a low-pass, eight-pole Bessel filter at $30 \mathrm{~Hz}$ and digitized.

The suction electrode was filled with a solution containing (in $\mathrm{mm}$ ): $134.5 \mathrm{Na}^{+}, 3.6 \mathrm{~K}^{+}, 2.4 \mathrm{Mg}^{2+}, 1.2 \mathrm{Ca}^{2+}, 136.3 \mathrm{Cl}^{-}, 3$ succinate, 3 L-glutamate, 10 glucose, 10 HEPES, and 0.02 EDTA plus basal medium Eagle (BME) amino acid supplement and BME vitamin supplement (Life Technologies). The perfusion medium was the same except that 20 mM $\mathrm{NaHCO}_{3}$ replaced an equimolar amount of $\mathrm{NaCl}$. The optical bench design was as previously described (Baylor et al., 1979a). Unpolarized 8 msec flashes at $500 \mathrm{~nm}(10 \mathrm{~nm}$ bandwidth) were used for stimulation throughout.

\section{RESULTS}

\section{Generation of GC-E-deficient animals}

The mouse GC-E gene (Gucy2e) was disrupted by replacing a portion of exon 5 , which codes for the transmembrane region, with a neomycin-resistance gene cassette (Fig. 1a; see Materials and Methods). The targeted allele was maintained in both 129/ SvJ and C57BL/6J backgrounds. The homozygous null animals (Fig. $1 b$ ) were fully viable and fertile, indicating that GC-E is not required for normal development. Moreover, there were no noticeable abnormalities in the appearance or behavior of the knock-out animals when they were compared with their wild-type and heterozygous littermates in the normal animal-housing environment.

Western-blot analysis was performed to confirm the absence of GC-E expression in the knock-out animals. The posterior portion of the eyecup was homogenized in Laemmli buffer, and total proteins were separated by SDS-PAGE. Proteins were transferred to PVDF membranes and blotted with an antiserum specific to the C-terminal portion of GC-E (Yang and Garbers, 1997). This confirmed that the mutant allele eliminates the expression of GC-E (Fig. 1c). The expression level of GC-F was unchanged in 10-week-old homozygous null mice, as was the level of $r d s /$ peripherin, an integral membrane glycoprotein located in photoreceptor outer segment disks (Connell et al., 1991; Travis et al., 1991) (Fig. $1 c$ ). Even at 6 months of age the levels of GC-F, $r d s /$ peripherin, and rhodopsin were comparable in wild-type and null animals (data not shown), suggesting that the rod outer segments remained mainly intact in the null animals.

\section{Retinal structure of GC-E-deficient mice}

Under light microscopy the development of the outer segment layer in animals 1-3 weeks of age appeared normal in GC-E null 
a
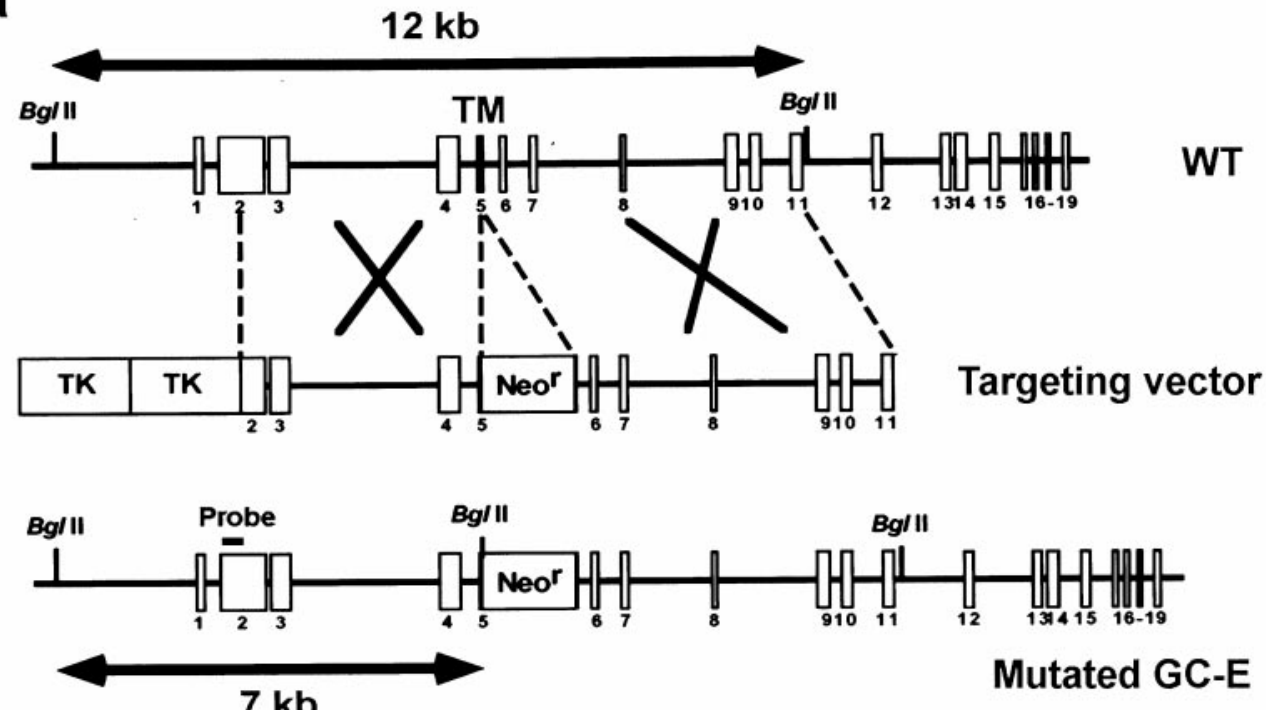

Figure 1. Targeted disruption of the GC-E gene in mice. $a$, Maps of the wild-type GC-E locus, the targeting vector, and the mutated locus. The coding exons of the GC-E gene are shown as open boxes. The map of the targeting vector shows the replacement of a portion of exon 5 coding for the transmembrane region with the neomycinresistance gene $\left(\mathrm{NeO}^{r}\right)$. Two copies of the thymidine kinase $(T K)$ gene were placed at the 5 '-end of the targeting vector for negative selection. The expected sizes of $B g l$ III-generated fragments from the wild-type and disrupted GC-E genes detected with a $5^{\prime}$-flanking probe (bold bar) are shown. $b$, Southern blot analysis of DNA from ES cell clones and tail DNA from littermates. The appearance of a $7 \mathrm{~kb}$ band from the mutant allele is indicated. $c$, Western blot analysis of retinal extracts from 10-week-old wild-type $(+/+)$ and homozygous null (-/-) mice. Anti-GC-E, GC-F (Yang and Garbers, 1997), and $r d s /$ peripherin antisera (Travis et al., 1991) were used for immunodetection.

animals. The overall retinal structure continued to appear normal up to 1 year, with unaltered thickness of the various retinal layers (Fig. 2). The number of photoreceptor nuclei was counted from retinal sections from two wild-type and two null animals. At 12 months of age the wild-type retina contained $151 \pm 12$ photoreceptor nuclei $/ 2000 \mu \mathrm{m}^{2}$, whereas the GC-E - /- retina contained $140 \pm 12$ photoreceptor nuclei/2000 $\mu \mathrm{m}^{2}$ (mean \pm SEM). The retinas of heterozygous animals likewise appeared morphologically normal at all ages (data not shown).

Although with light microscopy the outer segments of cone cells cannot be identified in the rod-dominant mouse retina, these cells can be distinguished by their nuclear morphology. Cone cell bodies can be recognized on the basis of their oval shape containing one to three clumps of chromatin and a large amount of lightly staining euchromatin; rods, on the other hand, have a round nucleus containing a single clump of chromatin with little euchromatin (Carter-Dawson and LaVail, 1979). At 4 weeks of age, the earliest age to identify reliably the cone cell nuclei by this method, the number of cone cell bodies is similar in wild-type and null animals. By 5 weeks of age, however, the number of identifiable cones has been reduced dramatically in the null mice, with only an occasional cone cell body recognizable in the outer nuclear layer (Fig. $3 a-d$ ). At later ages the number of cone cells appears to remain stable, suggesting the continued survival of the cones that were present at 5 weeks of age. Thus, although the lack of GC-E does not seem to affect the differentiation of cones, these cells degenerate rapidly at 4-5 weeks of age, with a few cones surviving at late ages. The fact that the overall thickness of the outer nuclear layer does not noticeably change despite cone degeneration again suggests that the rods remain relatively intact.

The lectin PNA has been shown to label specifically the cone cell outer and inner segments in several species, including mouse (Blanks and Johnson, 1984). We used this lectin to label cone cells in wild-type and GC-E knock-out retinas to confirm further the time course of cone cell loss in the knock-out mice. At the age of 4 weeks the labeling of scattered photoreceptor outer and inner segments, which are presumably cones, was observed in both wild-type and null animals (Fig. 3e,f). Cone inner segments were stained the most strongly. Equivalent labeling of wild-type and null animals also was observed in 3-week-old animals (data not shown). The specificity of PNA binding was confirmed by competitive inhibition of binding by $50 \mathrm{~mm}$ D-galactose (data not shown). No PNA label was present in the retina of 5-week-old GC-E null mice, although labeling was unchanged in wild-type animals (Fig. $3 g, h$ ). This confirmed the disappearance of cone cell nuclei that we had observed in sections stained with hematoxylin and eosin.

\section{ERG measurements}

To examine whether the loss of GC-E affects retinal function, we measured full-field ERGs in wild-type, heterozygous, and null animals. The ERGs of GC-E null animals showed alterations as early as 1 month of age (Fig. 4a, right column), with the rod a-wave (top panel) and b-wave (middle panel) both markedly reduced. Cone responses to white flashes in the presence of a rodsaturating background (bottom panel) were barely detectable. 


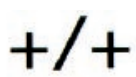

a

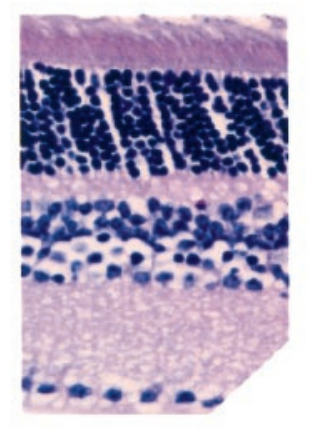

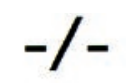

b

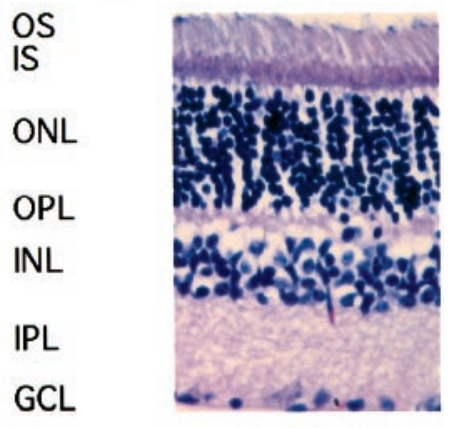

C

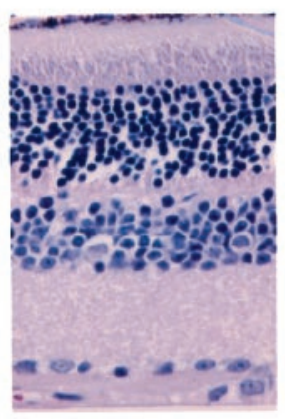

IS

ONL

OPL

INL

IPL

GCL

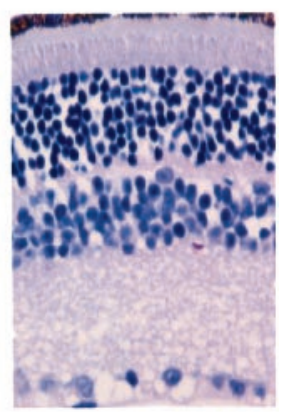

Figure 2. Retinal morphology of wild-type $(a, c)$ and GC-E knock-out mice $(b, d)$ at 10 weeks $(a, b)$ and 12 months $(c, d)$ of age. Shown are light micrographs of $4 \mu \mathrm{m}$ sections cut through the optic nerve head stained with hematoxylin and eosin. Magnification, $100 \times . O S$, Outer segment; $I S$, inner segment; $O N L$, outer nuclear layer; $O P L$, outer plexiform layer; $I N L$, inner nuclear layer; $I P L$, inner plexiform layer; $G C L$, ganglion cell layer.

Interestingly, this reduction in ERG response was evident before the apparent disappearance of cone cells, which occurred $\sim 5$ weeks of age. At 5 months of age (Fig. 4b) there was no further decrease in rod ERG amplitude, but the cone ERG was nondetectable and, in fact, was absent as early as 2 months of age (data not shown). Values for the sensitivity parameter $(S)$ and the maximal amplitude $(R m P 3)$ of the rod response were calculated for each animal by fitting Equation 1 (see Materials and Methods) to the leading edge of the rod a-wave (dashed curves in Fig. 4a, $b$, top panel). The values for $S$, which reflects the amplification of the activation phase of phototransduction, were comparable between knock-out and wild-type animals at all ages. However, $R m P 3$ was consistently lower for GC-E -/- mice up to 1 year of age (Fig. $4 c$ ). By comparison, the ERG responses of heterozygous mice were normal up to 1 year of age (data not shown). The reduced a-wave suggests that rod function is compromised in the null animals despite their apparently normal morphology. The reduction of the b-wave, which is approximately proportional to the a-wave reduction, is not surprising because it arises primarily from the electrical activity of secondary neurons (Ogden, 1994) and therefore is influenced by the a-wave. Finally, the disappearance of the cone response is consistent with the morphological finding that the number of cone cells is reduced sharply in animals that are older than 5 weeks.

Double-flash experiments were performed to determine whether recovery from activation was altered in GC-E null animals. In this protocol the recovery of the underlying photoresponse to a test flash was probed with a second intense flash that followed the test flash at various times (interflash intervals). As shown in Figure $4 d$, the derived photoresponse of a representative GC-E - / - animal begins to decline from saturation sooner than that of a wild-type animal. The mean period of saturation of the a-wave (parameter T, Eq. 2 in Materials and Methods) at the intensity used for the first flash was significantly shorter $(p<$ $0.001 ; t$ test $)$ in the null animals $(205 \pm 16 \mathrm{msec}$; mean $\pm \mathrm{SEM}$; $n=6)$ than in wild-type animals (372 $\pm 32 \mathrm{msec} ; n=8)$.

\section{Rod photoreceptor electrophysiology}

The reduction in the ERG a-wave in GC-E null animals prompted us to examine the impact of the absence of GC-E on rod function. Responses of single dark-adapted rods, either isolated or projecting from fragments of mechanically dissociated retina, were recorded with a suction pipette (Baylor et al., 1979a) (see Materials and Methods). In contrast to the reduction in the ERG a-wave amplitude, the saturated response elicited by a bright flash from rods of null animals was normal in amplitude ( $r_{\text {max }}$, Table 1), suggesting that the dark current through the cGMP-activated channels was approximately unchanged. There were changes in other respects, however. Although the flash response of a rod from knock-out animals rose with normal kinetics, the response exhibited an increased time-to-peak $\left(t_{\mathrm{p}}\right.$, Table 1) before decaying more rapidly than normal (compare Fig. $5 a$ with $b$ ). The rods from null mice also show oscillations during and after recovery from a light flash; the reason for this remains unclear. Such oscillations have been demonstrated previously in calculations under conditions in which $\mathrm{Ca}^{2+}$ buffering is increased (Nikonov et al., 1998). Additionally, after the light flash the wild-type mice demonstrate multiple apparent phases of recovery (Fig. $5 a$ ), whereas the null mice show predominantly one phase (Fig. $5 b$ ). The longer time-to-peak with the same trajectory led to a higher sensitivity of the GC-E null rods, which can be seen from the flash intensity-response relations in Figure $5 c$ (also $I_{\mathrm{o}}$, Table 1). This increase in the flash sensitivity would not be detected by the ERG measurements because the b-wave intrudes and truncates the a-wave (see Fig. 4a, top panel). The higher sensitivity of rods from mutant mice also was indicated by the larger amplitude of the single-photon response ( $a$, Table 1), calculated from the variance/mean ratio of the response amplitudes of the cell to repetitive, identical dim flashes (see Baylor et al., 1979b). These results indicate that there is a disparity between ERG and single-rod recordings with respect to the rod response amplitude; however, with both techniques a faster recovery of rods from light is apparent.

\section{DISCUSSION}

A severe reduction in cones and a vanishing cone response to light as a function of age represent the dominant phenotype of GC-E null mice. However, such mice also display a decreased rod response to light, based on ERG recordings. The marked decrease in the ERG response occurs despite the morphologically normal appearance of the rods. The likeliest consequence of the absence of GC-E is a reduction of the cGMP level in cones in both dark and light conditions. This decrease itself could facilitate degeneration, or alternatively, the cessation of the dark current could be the primary effector of cone loss. Chronic light exposure can cause degeneration as well, possibly via mechanisms similar 

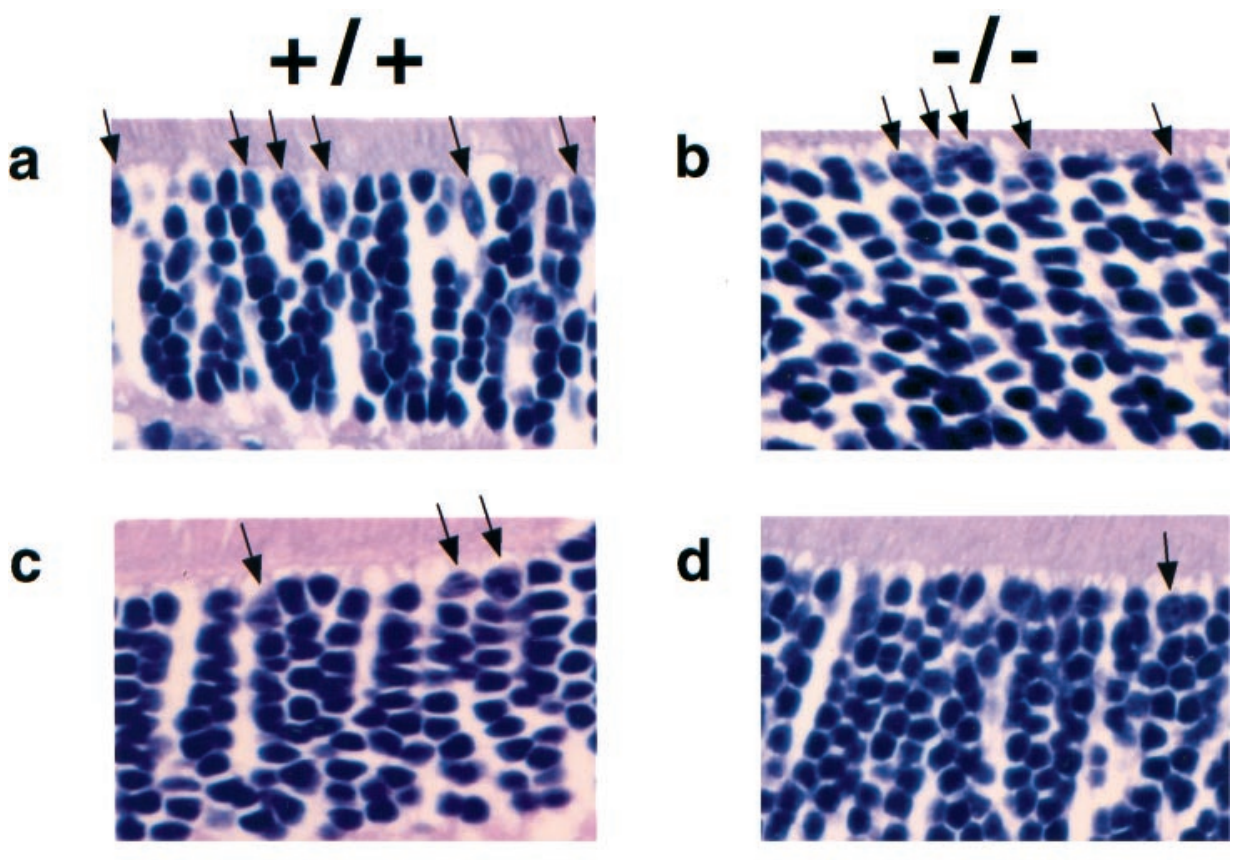

e
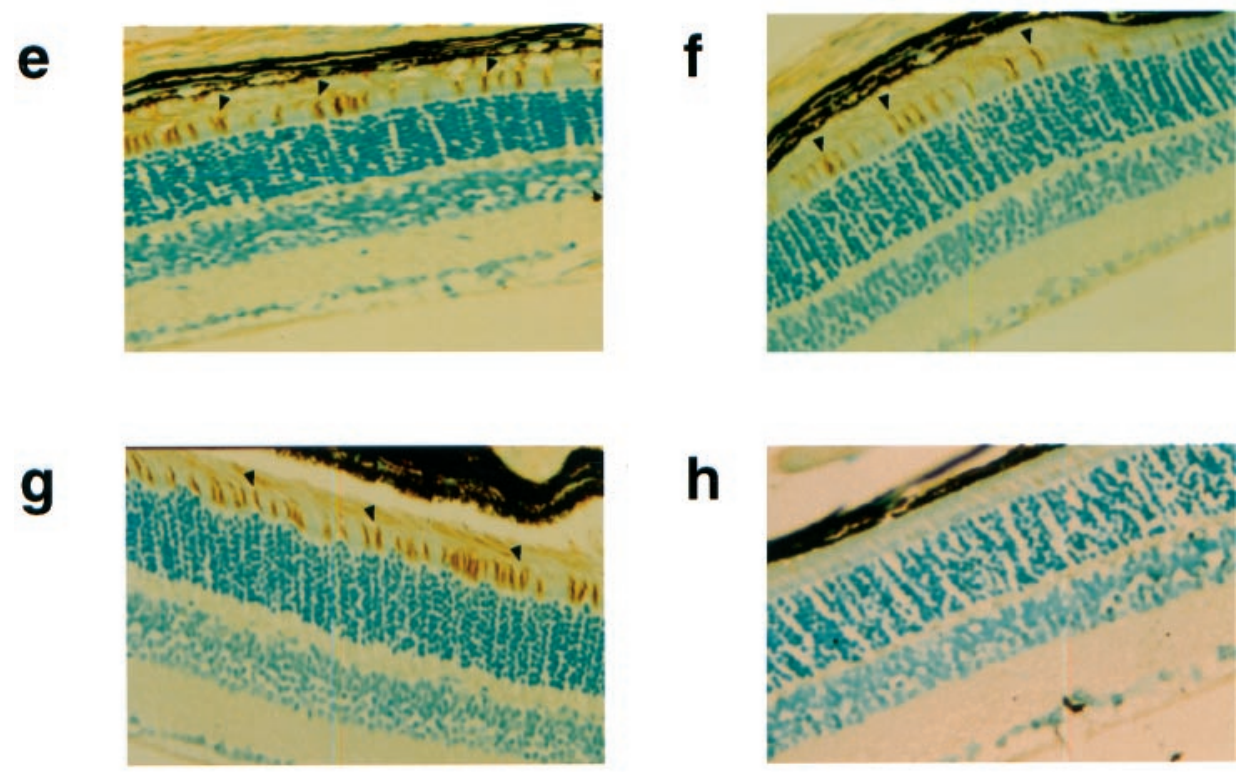

Figure 3. Cone photoreceptors are lost between 4 and 5 weeks of age in GC-E knockout mice. Left column, wild-type animals $(+/+)$; right column, GC-E null animals $(-/-)$. Shown are light micrographs of $4-\mu \mathrm{m}-$ thick hematoxylin- and eosin-stained sections from animals of 4 weeks $(a, b)$ and 5 weeks $(c$, $d$ ) of age. Few cone cell nuclei are detectable in $-/-$ mice at 5 weeks of age. However, cone cell nuclei are clearly evident in wild-type littermates (arrows). Magnification, 250×. Also shown are peanut agglutinin labeling of retinas from 4-week-old $(e, f)$ and 5-week-old $(g, h)$ mice. Cone photoreceptors (arrowheads) are present in equal numbers in 4-week-old wild-type and null animals and 5-week-old wild-type mice, but they are not present in 5-week-old GC-E -/- mice. Magnification, $100 \times$. to that induced by the elimination of GC-E (Fain and Lisman, 1993). We do not know if null mice kept in the dark would retain cones in the absence of GC-E.

The a-wave of the dark-adapted ERG is much smaller in amplitude than normal for the null animals, suggesting a substantial loss of rod function, in part concomitant with cone degeneration. However, single-rod recordings suggest that the saturated response of individual rods is close to normal. Thus, the reason for the reduction in the a-wave is unclear. Possibly, because of the disappearance of cones and associated changes in the extracellular matrix, the transretinal resistance decreases. As a result, the same current response generated in rods would produce a smaller ERG a-wave. Even before the disappearance of cone cells at $\sim 5$ weeks of age, the functioning of these cells may be compromised, leading to alterations in retinal function despite apparently normal morphology as assessed with light microscopy. A similar disparity between the ERG a-wave and single-rod recordings has been reported previously for mice lacking the phospholipase $\mathrm{C} \beta 4$ enzyme normally present in rods (Jiang et al., 1996; Peng et al., 1997).

Despite a normal dark current the flash response of rods from null mice is aberrant. The response rises with kinetics similar to control but then has a longer time-to-peak, thus reaching higher amplitude for a given flash intensity (i.e., a larger single-photon response). These characteristics often are associated with negative-feedback control on phototransduction. An interruption of the intracellular $\mathrm{Ca}^{2+}$ decline, for example, could result in a light response rising unchecked via negative feedback (Yau, 1994). In this scenario, however, the flash response is expected to decline more slowly (Yau, 1994), opposite to the accelerated recovery kinetics observed in both ERG and single-rod recordings. Based on modeling, the peculiar response characteristics 

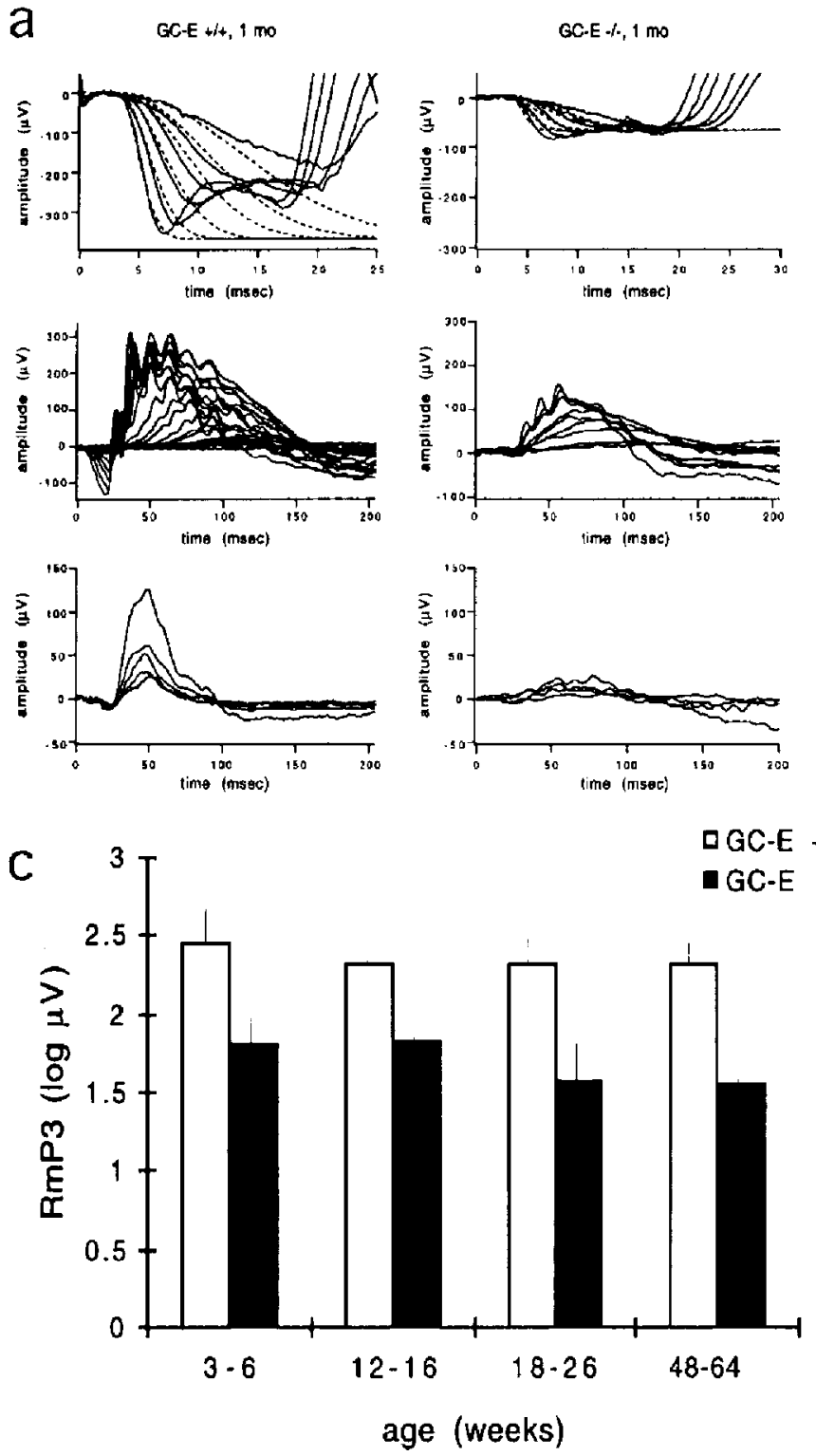

b

GC.E $+1+, 5$ mo
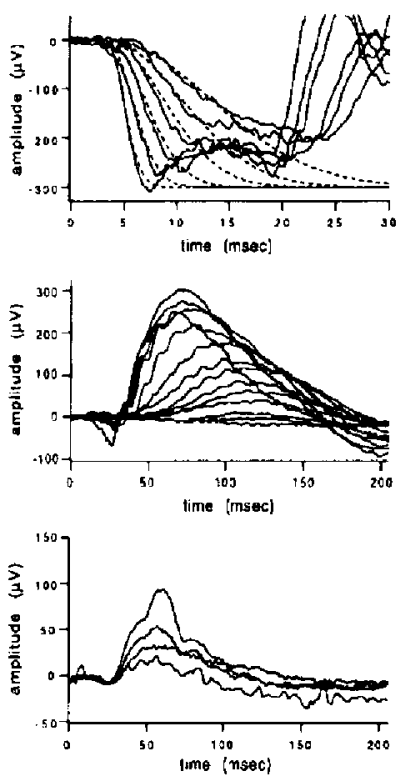

d
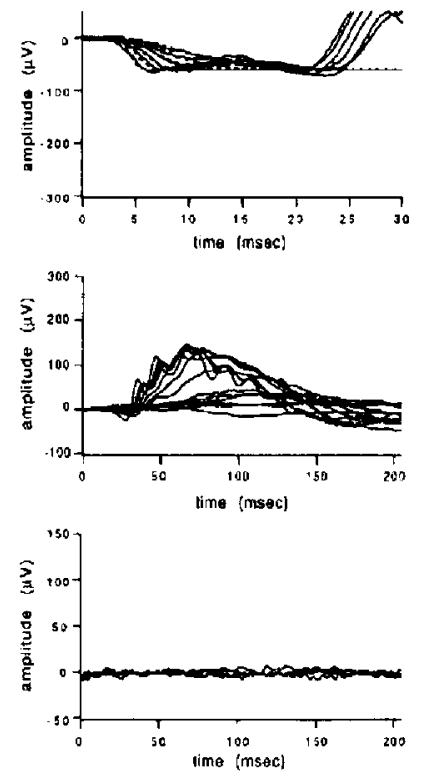

d

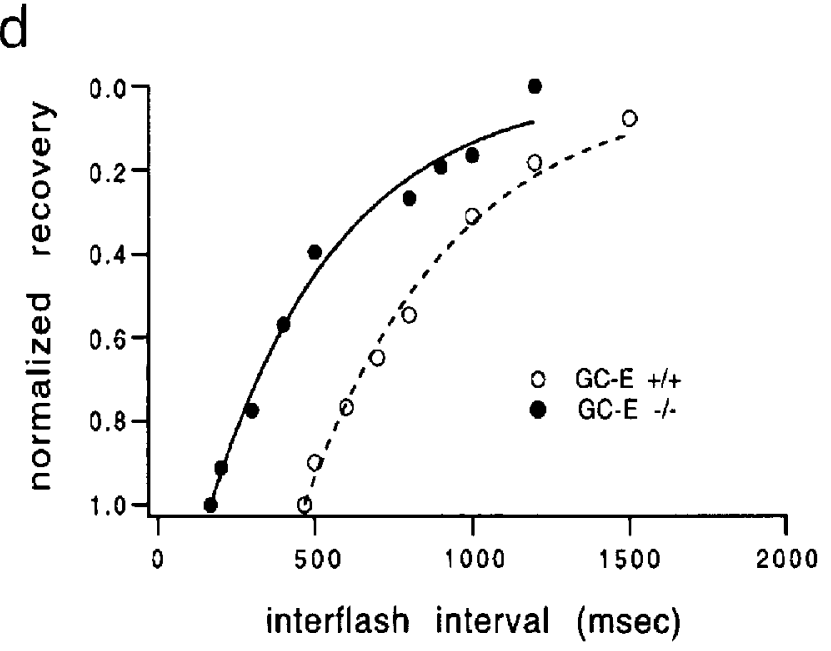

Figure 4. Full-field ERG responses from wild-type and GC-E-deficient mice. $a, b$, Top, Responses to short-wavelength flashes from 1.8 to 3.4 log scot td-sec in $0.3 \log$ unit steps. Dashed curves are fits of Equation 1 to the leading edge of the responses. Middle, Responses to short-wavelength stimuli ranging from -3 to $1 \log \operatorname{scot}$ td-sec $(0.3 \log$ unit steps). The b-wave is the main component. Bottom, Cone responses to white flashes from 0.24 to 1.44 $\log$ photopic troland-seconds $\left(0.3 \mathrm{log}\right.$ unit steps) in the presence of a rod-saturating $\left(40 \mathrm{~cd} / \mathrm{m}^{2}\right)$ background. No cone response is detectable in the $-/-$ animal at the age of 5 months. $c$, Plot of maximal amplitude of ERG a-wave (RmP3) with increasing age. Data are averaged (mean \pm SEM) from 20 wild-type and $17-/-$ animals. $d$, Decline of $R m P 3$ as a function of the interflash interval in a double-flash experiment (see Materials and Methods) from representative animals. The best-fit curves are exponential declines. The critical period $T$ is $208 \mathrm{msec}$ for the GC-E null mouse and $467 \mathrm{msec}$ for the wild-type mouse.

demonstrated by the GC-E null rods in principle can be explained by particular conditions with intracellular $\mathrm{Ca}^{2+}$ buffering $(\mathrm{Ni}-$ konov et al., 1998). However, no experiments to measure the effects of a lack of GC-E on photoreceptor $\mathrm{Ca}^{2+}$ have been performed yet.

What is the implication of an unchanged rod dark current in GC-E - /- mice? The simplest interpretation would be that GC-E is present insignificantly, if at all, in rods and therefore contributes little to cGMP synthesis, with this function being performed by GC-F. Alternatively, GC-E may be important for rod cGMP synthesis, but the dark current remains constant in null animals because there is a compensatory increase in the number of cGMP-activated channels on the plasma membrane or an increase in the affinity of the channels for cGMP. However, there is no evidence for such compensatory changes because the length of the rod outer segment of null animals appears normal, as do the activation properties of the channels and their density on the plasma membrane (data not shown). Another potential compensation for the lack of GC-E is an increase in the activity of GC-F. Although GC-F expression is unchanged (see Results), its catalytic activity could increase if the inhibition by $\mathrm{Ca}^{2+}$ is changed, possibly because of a decrease in the free $\mathrm{Ca}^{2+}$ concentration in the mutant rods in darkness. However, this is not expected, given that the free $\mathrm{Ca}^{2+}$ concentration in the steady 
Table 1. Parameters for flash responses of rods from wild-type and GC-E null mice

\begin{tabular}{lcc} 
Parameter & Wild-type & GC-E $-/-$ \\
\hline$t_{\mathrm{p}}(\mathrm{msec})$ & $152.0 \pm 11.3(26)$ & $203.1 \pm 7.5(41)^{a}$ \\
$t_{\mathrm{i}}(\mathrm{msec})$ & $279.6 \pm 20.9(26)$ & $216.6 \pm 14.4(41)^{a}$ \\
$a(\mathrm{pA})$ & $0.41 \pm 0.03(26)$ & $0.51 \pm 0.02(41)^{a}$ \\
$I_{\mathrm{o}}\left(\mathrm{photons} / \mu \mathrm{m}^{2}\right)$ & $68.2 \pm 2.8(26)$ & $30.8 \pm 1.8(35)^{a}$ \\
$r_{\max }(\mathrm{pA})$ & $8.4 \pm 0.5(40)$ & $8.0 \pm 0.3(58)$
\end{tabular}

Values are mean \pm SEM; the number in parentheses indicates the number of rods tested. $t_{\mathrm{p}}$, Time-to-peak of the dim-flash response; $t_{\mathrm{i}}$, integration time of the dimflash response (see Baylor et al. 1979a); $a$, amplitude of the single-photon response; $I_{\mathrm{o}}$, flash strength at $500 \mathrm{~nm}$, which gave rise to the half-maximal response; $r_{\text {max }}$, saturating photoresponse amplitude.

${ }^{a}$ Significantly different from control value ( $p<0.05$; independent $t$ test).

state should depend only on the dark current and the $\mathrm{Ca}^{2+}$ efflux through the $\mathrm{Na}^{+} / \mathrm{Ca}^{2+}, \mathrm{K}^{+}$exchanger (Yau, 1994). In other words, with the dark current near normal and the properties of the exchanger presumably unchanged, the free $\mathrm{Ca}^{2+}$ concentration should stay constant, unless a minor difference in the dark current is able to change the feedback control on GC-F dramatically.

The gene for GC-F is located in region $\mathrm{Xq} 22$ of the human $\mathrm{X}$ chromosome (Yang et al., 1996), to which no retinal diseases have been mapped. The GC-E gene is located in mouse chromosome 11, syntenic with human chromosome 17p13.1 (Oliveira et al., 1994; Yang et al., 1996), which has been linked with several retinopathies (Joshi et al., 1997). Recently, mutations in GC-E have been identified in human retinal diseases. Missense and frame-shift mutations in the human GC-E gene that are expected to render the protein nonfunctional have been associated with Leber congenital amaurosis, characterized by early-onset and widespread rod/cone degeneration (Perrault et al., 1996). Like Leber's disease, the mouse $\mathrm{GC}-\mathrm{E}$ null phenotype is recessive; unlike the disease, however, the mouse phenotype consists pri- marily of cone degeneration. One might argue that, unlike the rod-dominant mouse retina, the human retina has substantially more cones so that cone degeneration would disrupt the integrity of the human retina more severely and consequently cause concomitant rod degeneration. Other missense mutations of the human GC-E gene lead to a form of cone/rod dystrophy (CORD6), a disease that initially affects cones, followed by rod degeneration later (Kelsell et al., 1998; Perrault et al., 1998). Although CORD6 resembles the mouse GC-E null phenotype, the human disease is hereditarily dominant. Furthermore, the GC-E missense mutations associated with CORD6 are thought to affect dimerization of the enzyme rather than to cause its complete elimination, as in the knock-out. Finally, in the chicken a naturally occurring null mutation of the orthologous GC-E gene has been found to cause autosomal recessive retinal degeneration (Ulshafer et al., 1984; Semple-Rowland et al., 1998). Although the degeneration in the $r d$ chicken apparently differs from that in the GC-E null mouse by affecting both rods and cones, the difference in this case again could be explained by the chicken retina being cone-rich (having a cone/rod ratio much higher than in the mouse retina).

Another complexity that should be considered in interpreting our results and the disease phenotypes has to do with GCAP1 and GCAP2, the $\mathrm{Ca}^{2+}$-binding proteins that regulate $\mathrm{GC}-\mathrm{E}$ and GC-F function. There appears to be uniform agreement, based on immunocytochemistry, that GCAP1 is present in both rod and cone outer segments (Palczewski et al., 1994; Frins et al., 1996; Cuenca et al., 1998; Howes et al., 1998). The localization of GCAP2 is more controversial and perhaps species-dependent. It may be expressed in both rod and cone outer segments (OttoBruc et al., 1997; Cuenca et al., 1998; Howes et al., 1998), although biochemical experiments suggest that GCAP1 and not GCAP2 is the major stimulator of guanylyl cyclase in rod outer segments (Otto-Bruc et al., 1997). It was demonstrated recently that a missense mutation in the human GCAP1 gene leads to autosomal dominant cone dystrophy (Payne et al., 1998), appar-

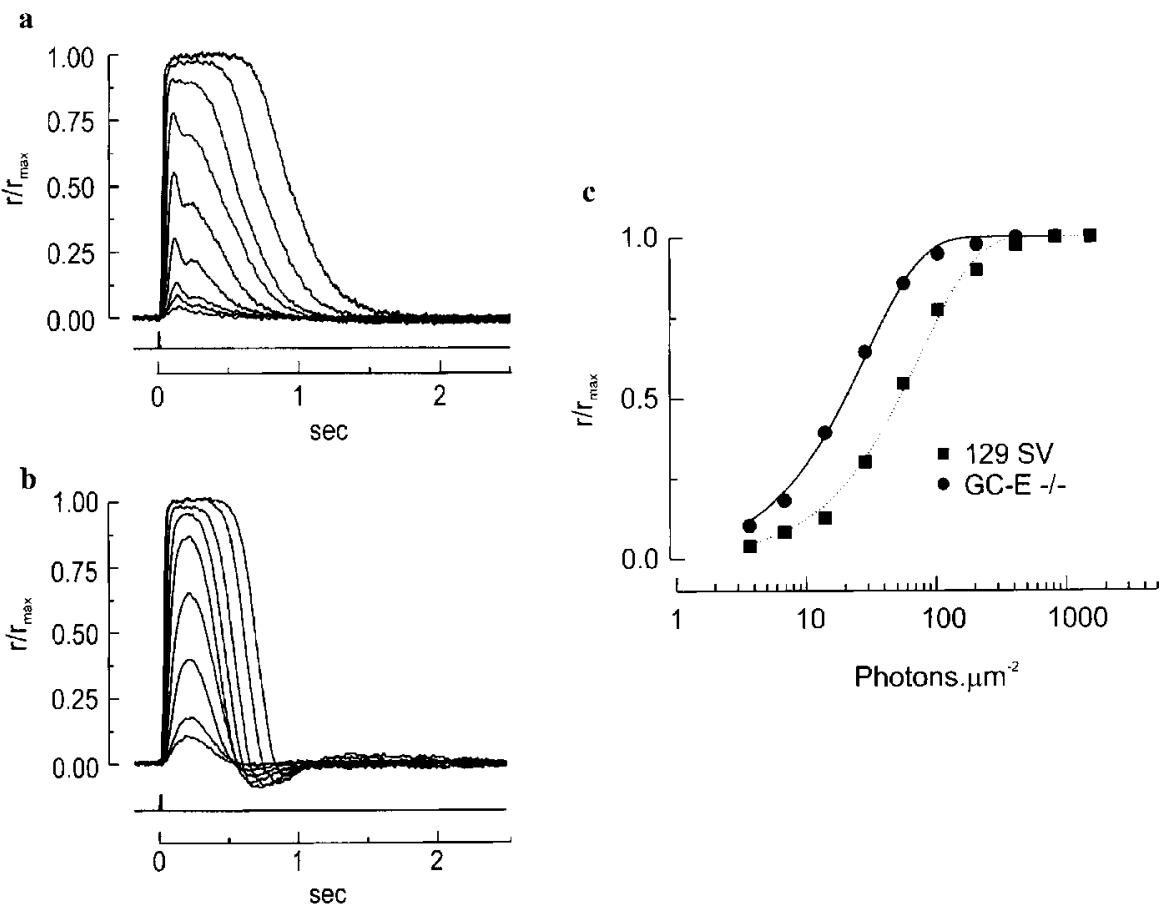

Figure 5. Suction pipette recordings from single rods of wild-type and GC-E null mice. $a, b$, Normalized responses of a rod from a wild-type mouse (a) and a GC-E - / - mouse (b) to $500 \mathrm{~nm}$ flashes of increasing strength. Each trace is the averaged response from multiple flash trials. The records were low-pass-filtered at $30 \mathrm{~Hz}$. Flash monitor output is shown by the bottom trace in each panel. The maximal response for $a$ was $14.3 \mathrm{pA}$ and for $b$ was 10.7 pA. $c$, Relation between the peak amplitude of flash response and the flash intensity for the two rods shown in $a$ and $b$. Wild-type, squares; GC-E $-/-$, circles. Curves are fit with the exponential saturation function, $r / r_{\max }=1-\exp (-k i)$, where $k$ is a constant inversely proportional to the sensitivity of the cell and $i$ is the flash strength. Halfmaximal responses occurred at 53 photons $/ \mu \mathrm{m}^{2}$ for the wild-type rod and 20 photons $/ \mu \mathrm{m}^{2}$ for the GC-E $-/-$ rod. 
ently because of constitutive activation of guanylyl cyclase (Dizhoor et al., 1998; Sokal et al., 1998). The degeneration of cones and the sparing of rods suggest that GCAP1 is functionally important in cones but perhaps not as critical in rods. The observation of a similar phenotype, i.e., cone degeneration, when GC-E or GCAP1 is mutated suggests that tight regulation of guanylyl cyclase activity in these cells may be crucial for their survival.

The GC-E-deficient mouse demonstrates that this protein is essential for normal retinal structure and function. It appears that $\mathrm{GC}-\mathrm{E}$ is required for the survival of cone photoreceptors at an apparently critical period at the age of $\sim 5$ weeks, whereas rod photoreceptors appear to remain normal even at advanced ages. Although the kinetics of activation of phototransduction is unchanged in rods, the maximum ERG response is reduced markedly, and recovery after a light flash is faster in mice lacking GC-E. These results suggest an important role for this guanylyl cyclase in both the survival of a subclass of retinal cells and in the delicate balance of cellular processes that lead to vision.

\section{REFERENCES}

Baylor DA, Lamb TD, Yau K-W (1979a) The membrane current of single rod outer segments. J Physiol (Lond) 288:589-611.

Baylor DA, Lamb TD, Yau K-W (1979b) Responses of retinal rods to single photons. J Physiol (Lond) 288:613-634.

Blanks JC, Johnson LV (1984) Specific binding of peanut lectin to a class of retinal photoreceptor cells: a species comparison. Invest Ophthalmol Vis Sci 25:546-557.

Carter-Dawson LD, LaVail MM (1979) Rods and cones in the mouse retina. I. Structural analysis using light and electron microscopy. J Comp Neurol 188:245-262.

Connell G, Bascom R, Molday L, Reid D, McInnes RR, Molday RS (1991) Photoreceptor peripherin is the normal product of the gene responsible for retinal degeneration in the $r d s$ mouse. Proc Natl Acad Sci USA 88:723-726.

Cooper N, Liu L, Yoshida A, Pozdnyakov N, Margulis A, Sitaramayya A (1995) The bovine rod outer segment guanylate cyclase, ROS-GC, is present in both outer segment and synaptic layers of the retina. J Mol Neurosci 6:211-222.

Cuenca N, Lopez S, Howes K, Kolb H (1998) The localization of guanylyl cyclase-activating proteins in the mammalian retina. Invest Ophthalmol Vis Sci 39:1243-1250.

Dizhoor AM, Lowe DG, Olshevskaya EV, Laura RP, Hurley JB (1994) The human photoreceptor membrane guanylyl cyclase, RetGC, is present in outer segments and is regulated by calcium and a soluble activator. Neuron 12:1345-1352.

Dizhoor AM, Olshevskaya EV, Henzel WJ, Wong SC, Stults JT, Ankoudinova I, Hurley JB (1995) Cloning, sequencing, and expression of a $24 \mathrm{kDa} \mathrm{Ca}^{2+}$-binding protein activating photoreceptor guanylyl cyclase. J Biol Chem 270:25200-25206.

Dizhoor AM, Boikov SG, Olshevskaya EV (1998) Constitutive activation of photoreceptor guanylate cyclase by Y99C mutant of GCAP-1. Possible role in causing human autosomal dominant cone degeneration. J Biol Chem 273:17311-17314.

Fain GL, Lisman JE (1993) Photoreceptor degeneration in vitamin A deprivation and retinitis pigmentosa: the equivalent light hypothesis. Exp Eye Res 57:335-340.

Frins S, Bönigk W, Müller F, Kellner R, Koch K-W (1996) Functional characterization of a guanylyl cyclase-activating protein from vertebrate rods. Cloning, heterologous expression, and localization. J Biol Chem 271:8022-8027.

Garbers DL, Lowe DG (1994) Guanylyl cyclase receptors. J Biol Chem 269:30741-30744.

Howes K, Bronson JD, Dang YL, Li N, Zhang K, Ruiz C, Helekar B, Lee M, Subbaraya I, Kolb H, Chen J, Baehr W (1998) Gene array and expression of mouse retina guanylate cyclase-activating proteins 1 and 2. Invest Ophthalmol Vis Sci 39:867-875.

Jiang H, Lyubarsky A, Dodd R, Vardi N, Pugh E, Baylor D, Simon MI,
Wu D (1996) Phospholipase C $\beta 4$ is involved in modulating the visual response in mice. Proc Natl Acad Sci USA 93:14598-14601.

Joshi AR, Mullen L, Small KW (1997) The retina: genetic studies of several retinopathies located on the short arm of chromosome 17. Curr Opin Neurol 10:31-35.

Kelsell RE, Gregory-Evans K, Payne AM, Perrault I, Kaplan J, Yang R-B, Garbers DL, Bird AC, Moore AY, Hunt DM (1998) Mutations in the retinal guanylate cyclase $(R E T G C-1)$ gene in dominant cone-rod dystrophy. Hum Mol Genet 7:1179-1184.

Lagnado L, Baylor D (1992) Signal flow in visual signal transduction. Neuron 8:995-1002.

Lamb TD, Pugh EN (1992) A quantitative account of the activation steps involved in phototransduction in amphibian photoreceptors. J Physiol (Lond) 449:719-758.

Liu X, Seno K, Nishizawa Y, Hayashi F, Yamazaki A, Matsumoto H, Wakabayashi T, Usukura J (1994) Ultrastructural localization of retinal guanylate cyclase in human and monkey retinas. Exp Eye Res 59:761-768.

Lowe DG, Dizhoor AM, Liu K, Gu Q, Spencer M, Laura R, Lu L, Hurley JB (1995) Cloning and expression of a second photoreceptor-specific membrane retina guanylyl cyclase (RetGC), RetGC-2. Proc Natl Acad Sci USA 92:5535-5539.

Nikonov S, Engheta N, Pugh EN (1998) Kinetics of recovery of the dark-adapted salamander rod photoresponse. J Gen Physiol 111:7-37.

Ogden TE (1994) Clinical electrophysiology. In: Retina, 2nd Ed, Vol 1 (Ryan SJ, ed), pp 321-332. St. Louis: Mosby-Year Book.

Oliveira L, Miniou P, Viegas-Pequignot E, Rozet J-M, Dollfus H, Pittler SJ (1994) Human retinal guanylate cyclase $(G U C 2 D)$ maps to chromosome 17p13.1. Genomics 22:478-481.

Otto-Bruc A, Fariss RN, Haeseleer F, Huang J, Buczylko J, Surgucheva I, Baehr W, Milam AH, Palczewski K (1997) Localization of guanylate cyclase-activating protein 2 in mammalian retinas. Proc Natl Acad Sci USA 94:4727-4732.

Palczewski K, Subbaraya I, Gorczyca WA, Helekar BS, Ruiz CC, Ohguro H, Huang J, Zhao X, Crabb JW, Johnson RS, Walsh KA, Gray-Keller MP, Detwiler PB, Baehr W (1994) Molecular cloning and characterization of retinal photoreceptor guanylate cyclase-activating protein. Neuron 13:395-404.

Payne AM, Downes SM, Bessant DAR, Taylor R, Holder GE, Warren MJ, Bird AC, Bhattacharya SS (1998) A mutation in guanylate cyclase activator 1A $(G U C A 1 A)$ in an autosomal dominant cone dystrophy pedigree mapping to a new locus on chromosome 6p21.1. Hum Mol Genet 7:273-277.

Peng Y-W, Rhee SG, Yu W-P, Ho Y-K, Schoen T, Chader GJ, Yau K-W (1997) Identification of components of a phosphoinositide signaling pathway in retinal rod outer segments. Proc Natl Acad Sci USA 94:1995-2000.

Perrault I, Rozet JM, Calvas P, Gerber S, Camuzat A, Dollfus H, Châtelin S, Souied E, Ghazi I, Leowski C, Bonnemaison M, Le Paslier D, Frézal J, Dufier J-L, Pittler S, Munnich A, Kaplan J (1996) Retinalspecific guanylate cyclase gene mutations in Leber's congenital amaurosis. Nat Genet 14:461-464.

Perrault I, Rozet J-M, Gerber S, Kelsell RE, Souied E, Cabot A, Hunt DM, Munnich A, Kaplan J (1998) A retGC-1 mutation in autosomal dominant cone-rod dystrophy. Am J Hum Genet 63:651-654.

Polans A, Baehr W, Palczewski K (1996) Turned on by $\mathrm{Ca}^{2+}$ ! The physiology and pathology of $\mathrm{Ca}^{2+}$-binding proteins in the retina. Trends Neurosci 19:547-554

Ramírez-Solis R, Davis AC, Bradley A (1993) Gene targeting in embryonic stem cells. Methods Enzymol 225:855-878.

Seimiya M, Kusakabe T, Suzuki N (1997) Primary structure and differential gene expression of three membrane forms of guanylyl cyclase found in the eye of the teleost Oryzias latipes. J Biol Chem 272:23407-23417.

Semple-Rowland SL, Lee NR, van Hooser JP, Palczewski K, Baehr W (1998) A null mutation in the photoreceptor guanylate cyclase gene causes the retinal degeneration chicken phenotype. Proc Natl Acad Sci USA 95:1271-1276.

Shyjan AW, de Sauvage FJ, Gillett NA, Goeddel DV, Lowe DG (1992) Molecular cloning of a retina-specific membrane guanylyl cyclase. Neuron 9:727-737.

Sokal I, Li N, Surgucheva I, Warren MJ, Payne AM, Bhattacharya SS, 
Baehr W, Palczewski K (1998) GCAP1(Y99C) mutant is constitutively active in autosomal dominant cone dystrophy. Mol Cell 2:129-133.

Sung C-H, Makino C, Baylor D, Nathans J (1994) A rhodopsin gene mutation responsible for autosomal dominant retinitis pigmentosa results in a protein that is defective in localization to the photoreceptor outer segment. J Neurosci 14:5818-5833.

Travis GH, Sutcliffe JG, Bok D (1991) The retinal degeneration slow (rds) gene product is a photoreceptor disc membrane-associated glycoprotein. Neuron 6:61-70.

Ulshafer RJ, Allen C, Dawson WW, Wolf ED (1984) Hereditary retinal degeneration in the Rhode Island Red chicken. I. Histology and ERG. Exp Eye Res 39:125-135.

Yang R-B, Garbers DL (1997) Two eye guanylyl cyclases are expressed in the same photoreceptor cells and form homomers in preference to heteromers. J Biol Chem 272:13738-13742.

Yang R-B, Foster DC, Garbers DL, Fülle H-J (1995) Two membrane forms of guanylyl cyclase found in the eye. Proc Natl Acad Sci USA 92:602-606.

Yang R-B, Fülle H-J, Garbers DL (1996) Chromosomal localization and genomic organization of genes encoding guanylyl cyclase receptors expressed in olfactory sensory neurons and retina. Genomics $31: 367-372$.

Yarfitz S, Hurley JB (1994) Transduction mechanisms of vertebrate and invertebrate photoreceptors. J Biol Chem 269:14329-14332.

Yau K-W (1994) Phototransduction mechanism in retinal rods and cones. The Friedenwald lecture. Invest Ophthalmol Vis Sci 35:9-32. 